\title{
Viejas y \\ nuevas disparidades por género en el mercado laboral
}

Marcela Perticara, Ph.D. en Economía, Universidad de Texas. Académica FEN UAH Marcela Perticara, Ph.D. en Economia, Universidad de Texas. Académica FEN UAH
Mauricio Tejada, Ph.D. en Economia Universidad de Georgetown. Académico FEN UAH

América Latina es una de las regiones en el mundo con menores tasas de participación laboral femenina. Las mujeres no sólo están sub-representadas en el mercado laboral, sino que tambien reciben
salarios (en promedio) por debajo de los de los hombres. De acuerdo al Global Gender Gap Report 2014 publicado por el World Economic Forum (GGGR), la región ha logrado reducir - en la mayoría de los paises -de manera importante las brechas por género en salud, nero en el mercado laboral son aún grandes y persistentes en tiempo. Chile no es ajeno a esta tendencia: un reciente estudio de Comunidad Mujer sobre las disparidades por generero en los últimos años', pone en evidencia que estas diferencias se hacen notorias en el ámbito laboral. En particular en el pais existe una brecha salarial importante para las mujeres, en torno al $30-40 \%$ que se transmite y magnifica (por la influencia de las lagunas previsionales) a los montos de jubilaciones percibidas durante la vejez. ¿Cömo explicamos la persistencia de las distancias salariales entre hombres mujeres en el pais? ¿Es esto discriminación pura, o es producto de la composición del empleo en particular dónde se están insertando

las tasas de partic de hablar de salarios, repasar la evolución educativo y tramos de edad. En primer lugar, las tasas de participacion femenina en el pais han aumentado de manera consistente. Ta como se aprecia en el gráfico 1, para las personas sin ningún tipo de che

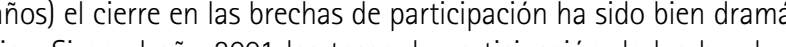
duplicaban las de las mujes hacia eño 2010 esta brecha hab cido a más de la mitad de este valor inicial para luego estabilizarse en el último quinquenio. Esta caida se debe tanto porque las mujeres aumentan fuertemente su participación (desde $41 \%$ a $58 \%$ en eriodo 2001-2015), como porque los hombres la reducen (desde 6\% a $90 \%$ en igual periodo). Para las personas más calificadas (con estudios superiores) la tendencia es menos pronunciada; las

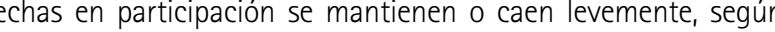
tramo elario. Patrones similares siguen las lasas de empleo, inmercado laboral ha

El cierre en la participación para el grupo menos educado, es acompañado de una reducción en la tasa de empleo independiente, lo que podria ser visto como una mejora en la calidad del empleo jeres más eduadas a fracción de nece casi constante, excepto para el grupo con 45-54 años de edad,

Lat rives salnial entre hombresy mujeres es en torno a 20-30\% para niveles educativos bajos (sin estudios superiores completos) tudios supucados, pero en torno a 40\% para mujeres con es-

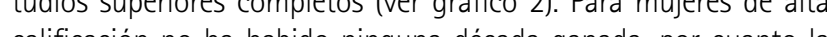
brecha es ta ala como na sido siempre. En linea con la literatura

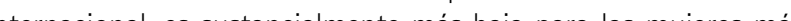
jóvenes (en torno a 20\%) pero casi se duplica para las mujeres en Jud tipicamente fentl para este grupo educativo (en torno a los 30 años). Para las mujeres sin calificación (sin ningún tipo de estudios superiores), si las diferencias salariales existentes a principios de los años 2000 eran del orden del 9\%-13\%, según el grupo etario, ahora se ubican ahora en torno al 20-30\%. Esto es, mientras este grupo de mujeres aumenta fuertemente su participación laboral y su tasa de empleo, pierde terreno en términos salariales.

¿Que hace que las mujeres se inserten laboralmente, pero reciban Una hipotesis que surge usualmente es que existe discriminación desfavorable hacia la mujer, que no necesariamente tiene que ser irracional, sino que puede suceder que en un pais la regulación haga particularmente cara a un ciento grupo demográfico y esto se traduzca en que los
salarios de este grupo

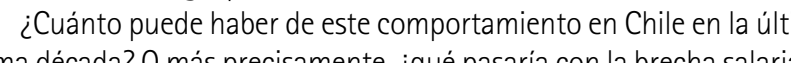
si se pudiera asilar el efecto puro de diferencias de productividad entre hombres y mujeres, de la discriminación y de la dinámica de mercado laboral (creación y destrucción de empleo)? El cuadro muestra los resultados del ejercicio de estimación que realizamos para responder estas preguntas para los años 2003 versus 2013, y para personas calificadas y no calificadas usando trabajadores asalariados². Algunas apreciaciones generales. Primero, la estimación confirma que la brecha para trabajadores calificados (salario de las mujeres sobre el salario de los hombres) se ha mantenido relativamente constante (ha caido levemente), mientras que la brecha para los trabajadores no calificados se ha más que duplicado, pasando de g\% a a brecha si la unica diferencia entre hombres y mujeres fuere producAqui se puede observar que para las mujes calificadas de altos ingresos a principal fuente de diferenciales salarias se atribuye diferencias en productividad. El componente discriminación es importante (un tercio de la brecha totall), pero no es lo único relevante Para las mujeres no calificadas, el aumento en la brecha salaria entre el año 2003 y 2013 se debe principalmente a diferenciales en productividad. No existe evidencia que exista discriminación pura por parte de los empleadores.
Es interesante evaluar brechas a lo largo de la distribución de salarios y no solo en el promedio. Si bien en el agregado (fila 4 ) no hay cambios, se ha desmejorado la productividad (y consiguientemente

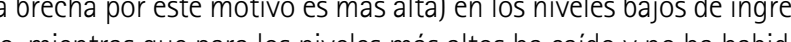
so mientras que para los niveles más allos ha caido y no ha habido no calficus la bres en su descomposicion. Pana los trabajadores y la principal fuente de diferenciales salariales son las diferenciales eroductividad

En resumen, para los trabajadores calificados (estudios superiores completos), las brechas en participación, empleo y salarios se mantienen relativamente constantes en los últimos años, con algunas fluctuaciones puntuales dependiendo del ciclo económico. Los cambios más dramáticos se dan para las mujeres de baja calificación, para las cuales las tasas de participación y empleo crecen de manera importante (entre 15-20 puntos porcentuales), a la vez que la diferencia de salarios se amplia de manera considerable (pasa de aproximadamente 10\% en los años 2000 a 30\% una décaen productividad, ia qué puede atribuirse? Al hablar de diferenciales de productividad no estamos diciendo que las mujeres que han ingresado sean menos productivas que los hombres, sino que se por tanto con menores salarios. En definitiva, la apertura del mercado laboral a las mujeres se ha dado en ciertas ocupaciones que no son las de mejores remuneraciones. Por tanto, las mujeres se están insertando en el mercado laboral pero en trabajos de muy baja productividad.

La conclusion de política pública con respecto a esta observación es que no es suficiente tener politicas de iqualdad salarial para igual puesto, sino que hay que generar instancias anteriores a la entrada a mercado laboral que garanticen a las mujeres mejores puestos de las mujeres no accedan a puestos de trabajo de altos (neferencias (aversión por ejemplo) y/o también de diseriminación (no salanial) por parte de los empleadores. El cambio cultural no se prese din para otro, pero ninguna pollica hacia el mundo empresaral funcionará si no educamos a nuestros jovenes (hombres y transicion, pollice como subsidios a b contraction de mujes en los tramos bajis y medios de calificacion y politicas de cuposen pustos de alta cilicación pueden acelerar los cam bios culturates a interior de las organizaciones que tanto se necesitan. 